\title{
The fundamental role of superconducting quasiparticle coherence in cuprate superconductors
}

\author{
Shiping Feng, Huaiming Guo \\ Department of Physics, Beijing Normal University, Beijing 100875, China
}

\begin{abstract}
Within the kinetic energy driven superconducting mechanism, we study the interplay between superconductivity and the nodal and antinodal superconducting quasiparticle coherences in cuprate superconductors, and find the s-wave superconducting transition temperature is heavily suppressed by the antinodal superconducting quasiparticle coherence, while the d-wave superconducting transition temperature is enhanced, therefore the antinodal superconducting quasiparticle coherence plays a more crucial role in superconductivity of cuprate superconductors.
\end{abstract}

Key words: Cuprate superconductors, Quasiparticle coherence, Superconducting mechanism PACS: 74.20.Mn, 74.25.Ha, 74.62.Dh

After twenty years intensive investigations, it has now become clear that cuprate superconductors are among the most complex systems studied in condensed matter physics [1]. These compounds have a layered structure of the square lattice of $\mathrm{CuO}_{2}$ plane separated by insulating layers, which leads to that cuprate superconductors are highly anisotropic materials, then the electronic excitation spectrum is dependent on the in-plane momentum $[2,3]$. The undoped cuprates are the Mott insulators with an antiferromagnetic (AF) long-range order (AFLRO), then upon the charge carrier doping, these compounds evolve into the superconductors leaving the $\mathrm{AF}$ short-range correlation still intact [1]. Experimentally, angle-resolved photoemission spectroscopy (ARPES) experiments have made a great deal of progress in the understanding of the underlying superconducting (SC) quasiparticle coherence of cuprate superconductors. On the one hand, superconductivity in doped cuprates results when electrons pair up into Cooper pairs [2] as in the conventional superconductors. Although the SC pairing mechanism is beyond the conven- tional electron-phonon mechanism, the SC-state of cuprate superconductors [4] is the conventional Bardeen-Cooper-Schrieffer (BCS) like, so that the basic BCS formalism is still valid in discussions of the electron excitation spectrum. In particular, the information revealed by ARPES experiments has shown that around the nodal and antinodal points of the Brillouin zone contain the essentials of the whole low energy quasiparticle excitation spectrum of cuprate superconductors $[2,3]$. On the other hand, unlike the conventional superconductors, the SC quasiparticle coherence plays an important role in superconductivity of doped cuprates [5]. Although both nodal and antinodal SC quasiparticle peaks exist for a wide range of the doping, the nodal and antinodal SC quasiparticle coherent weights tend to zero at the zero doping [2]. In this case, a natural question is whether of the nodal [6] and antinodal [5] SC quasiparticle coherences plays a more crucial role for superconductivity in doped cuprates.

Recently, we have developed a kinetic energy driven SC mechanism [7] based on the charge-spin separation (CSS) fermion-spin theory [8], where the 
dressed holons interact occurring directly through the kinetic energy by exchanging the spin excitations, leading to a net attractive force between the dressed holons, then the electron Cooper pairs originating from the dressed holon pairing state are due to the charge-spin recombination (CSR), and their condensation reveals the $\mathrm{SC}$ ground-state. In particular, this SC-state is controlled by both SC gap function and quasiparticle coherence, then the maximal SC transition temperature occurs around the optimal doping, and decreases in both underdoped and overdoped regimes [9]. In this paper, we discuss the interplay between superconductivity and the nodal and antinodal SC quasiparticle coherences in cuprate superconductors under this kinetic energy driven SC mechanism, and show that the s-wave $\mathrm{SC}$ transition temperature is heavily suppressed by the antinodal SC quasiparticle coherence, while the d-wave SC transition temperature is enhanced, therefore the antinodal SC quasiparticle coherence plays a more crucial role for superconductivity in doped cuprates.

Very soon after the discovery of superconductivity in doped cuprates, Anderson [10] suggested that the essential physics of doped cuprates is contained in the $t$ - $J$ model on a square lattice,

$$
\begin{aligned}
H & =-t \sum_{i \hat{\eta} \sigma} C_{i \sigma}^{\dagger} C_{i+\hat{\eta} \sigma}+t^{\prime} \sum_{i \hat{\tau} \sigma} C_{i \sigma}^{\dagger} C_{i+\hat{\tau} \sigma} \\
& +\mu \sum_{i \sigma} C_{i \sigma}^{\dagger} C_{i \sigma}+J \sum_{i \hat{\eta}} \mathbf{S}_{i} \cdot \mathbf{S}_{i+\hat{\eta}}
\end{aligned}
$$

where $\hat{\eta}= \pm \hat{x}, \pm \hat{y}, \hat{\tau}= \pm \hat{x} \pm \hat{y}, C_{i \sigma}^{\dagger}\left(C_{i \sigma}\right)$ is the electron creation (annihilation) operator, $\mathbf{S}_{i}=$ $C_{i}^{\dagger} \vec{\sigma} C_{i} / 2$ is spin operator with $\vec{\sigma}=\left(\sigma_{x}, \sigma_{y}, \sigma_{z}\right)$ as Pauli matrices, and $\mu$ is the chemical potential. This $t-J$ model is subject to an important local constraint $\sum_{\sigma} C_{i \sigma}^{\dagger} C_{i \sigma} \leq 1$ to avoid the double occupancy, which can be treated properly in analytical calculations within the CSS fermion-spin theory [8], where the constrained electron operators are decoupled as $C_{i \uparrow}=h_{i \uparrow}^{\dagger} S_{i}^{-}$and $C_{i \downarrow}=h_{i \downarrow}^{\dagger} S_{i}^{+}$, with the gauge invariant spinful fermion operator $h_{i \sigma}=$ $e^{-i \Phi_{i \sigma}} h_{i}$ describes the charge degree of freedom together with some effects of the spin configuration rearrangements due to the presence of the doped hole itself (dressed holon), while the spin operator $S_{i}$ describes the spin degree of freedom (spin), then the electron local constraint for the single occupancy is satisfied in analytical calculations. In this CSS fermion-spin representation, the low-energy behavior of the $t$ - $J$ model can be expressed as [11],

$$
\begin{aligned}
H & =t \sum_{i \hat{\eta}}\left(h_{i+\hat{\eta} \uparrow}^{\dagger} h_{i \uparrow} S_{i}^{+} S_{i+\hat{\eta}}^{-}+h_{i+\hat{\eta} \downarrow}^{\dagger} h_{i \downarrow} S_{i}^{-} S_{i+\hat{\eta}}^{+}\right) \\
& -t^{\prime} \sum_{i \hat{\tau}}\left(h_{i+\hat{\tau} \uparrow}^{\dagger} h_{i \uparrow} S_{i}^{+} S_{i+\hat{\tau}}^{-}+h_{i+\hat{\tau} \downarrow}^{\dagger} h_{i \downarrow} S_{i}^{-} S_{i+\hat{\tau}}^{+}\right) \\
& -\mu \sum_{i \sigma} h_{i \sigma}^{\dagger} h_{i \sigma}+J_{\mathrm{eff}} \sum_{i \hat{\eta}} \mathbf{S}_{i} \cdot \mathbf{S}_{i+\hat{\eta}}
\end{aligned}
$$

with $J_{\text {eff }}=(1-\delta)^{2} J$, and $\delta=\left\langle h_{i \sigma}^{\dagger} h_{i \sigma}\right\rangle=\left\langle h_{i}^{\dagger} h_{i}\right\rangle$ is the doping concentration.

Since ARPES measurements [12] have shown that in the real space the gap function and pairing force have a range of one lattice spacing, then the order parameter for the electron Cooper pair in the doped regime without AFLRO can be expressed as $[7,9], \Delta=\left\langle C_{i \uparrow}^{\dagger} C_{i+\hat{\eta} \downarrow}^{\dagger}-C_{i \downarrow}^{\dagger} C_{i+\hat{\eta} \uparrow}^{\dagger}\right\rangle=-\chi_{1} \Delta_{h}$, with the spin correlation function $\chi_{1}=\left\langle S_{i}^{+} S_{i+\hat{\eta}}^{-}\right\rangle$ and dressed holon pairing order parameter $\Delta_{h}=$ $\left\langle h_{i+\hat{\eta} \downarrow} h_{i \uparrow}-h_{i+\hat{\eta} \uparrow} h_{i \downarrow}\right\rangle$, which shows that the SC order parameter for the electron Cooper pair is related to the dressed holon pairing amplitude, and is proportional to the number of doped holes, and not to the number of electrons. In this case, we $[7,9]$ have shown within the Eliashberg's strong coupling theory [13] that the dressed holon-spin interaction can induce the dressed holon pairing state (then the electron Cooper pairing state) by exchanging the spin excitations in the higher power of the doping concentration, where the full dressed holon BCS type diagonal and off-diagonal Green's functions of the $t-J$ model (2) have been obtained as [11],

$$
\begin{gathered}
g(k)=Z_{h F}(\mathbf{k})\left(\frac{U_{h \mathbf{k}}^{2}}{i \omega_{n}-E_{h \mathbf{k}}}+\frac{V_{h \mathbf{k}}^{2}}{i \omega_{n}+E_{h \mathbf{k}}}\right), \\
\Im^{\dagger}(k)=-\frac{\bar{\Delta}_{h Z}(\mathbf{k})}{2 E_{h \mathbf{k}}}\left(\frac{Z_{h F}(\mathbf{k})}{i \omega_{n}-E_{h \mathbf{k}}}-\frac{Z_{h F}(\mathbf{k})}{i \omega_{n}+E_{h \mathbf{k}}}\right),
\end{gathered}
$$

respectively, where the four-vector notation $k=$ $\left(\mathbf{k}, i \omega_{n}\right), U_{h \mathbf{k}}^{2}=\left(1+\bar{\xi}_{\mathbf{k}} / E_{h \mathbf{k}}\right) / 2, V_{h \mathbf{k}}^{2}=(1-$ $\left.\bar{\xi}_{\mathbf{k}} / E_{h \mathbf{k}}\right) / 2, \bar{\Delta}_{h Z}(\mathbf{k})=Z_{h F}(\mathbf{k}) \bar{\Delta}_{h}(\mathbf{k}), \quad E_{h \mathbf{k}}=$ $\sqrt{\bar{\xi}_{\mathbf{k}}^{2}+\left|\bar{\Delta}_{h Z}(\mathbf{k})\right|^{2}}, \quad \overline{\xi_{\mathbf{k}}}=Z_{h F}(\mathbf{k})\left[\xi_{\mathbf{k}}+\Sigma_{1 e}^{(h)}(\mathbf{k})\right]$, while the static limit dressed holon effective gap function $\bar{\Delta}_{h}(\mathbf{k})=\left.\Sigma_{2}^{(h)}(\mathbf{k}, \omega)\right|_{\omega=0}$ and quasiparticle coherent weight $Z_{h F}^{-1}(\mathbf{k})=1-\left.\Sigma_{1 o}^{(h)}(\mathbf{k}, \omega)\right|_{\omega=0}$, with the dressed holon self-energy functions from the spin bubble $[9,11]$,

$$
\begin{aligned}
\Sigma_{1}^{(h)}(k) & =\frac{1}{N^{2}} \sum_{\mathbf{p}, \mathbf{p}^{\prime}}\left(Z t \gamma_{\mathbf{p}+\mathbf{p}^{\prime}+\mathbf{k}}-Z t^{\prime} \gamma_{\mathbf{p}+\mathbf{p}^{\prime}+\mathbf{k}}^{\prime}\right)^{2} \\
& \times \frac{1}{\beta} \sum_{i p_{m}} g(p+k) \Pi\left(\mathbf{p}^{\prime}, p\right)
\end{aligned}
$$




$$
\begin{aligned}
\Sigma_{2}^{(h)}(k) & =\frac{1}{N^{2}} \sum_{\mathbf{p}, \mathbf{p}^{\prime}}\left(Z t \gamma_{\mathbf{p}+\mathbf{p}^{\prime}+\mathbf{k}}-Z t^{\prime} \gamma_{\mathbf{p}+\mathbf{p}^{\prime}+\mathbf{k}}^{\prime}\right)^{2} \\
& \times \frac{1}{\beta} \sum_{i p_{m}} \Im(-p-k) \Pi\left(\mathbf{p}^{\prime}, p\right),
\end{aligned}
$$

where $\Pi\left(\mathbf{p}^{\prime}, p\right)=(1 / \beta) \sum_{i p_{m}^{\prime}} D^{(0)}\left(p^{\prime}\right) D^{(0)}\left(p^{\prime}+p\right)$, $\gamma_{\mathbf{k}}=(1 / Z) \sum_{\hat{\eta}} e^{i \mathbf{k} \cdot \hat{\eta}}, \gamma_{\mathbf{k}}^{\prime}=(1 / Z) \sum_{\hat{\tau}} e^{i \mathbf{k} \cdot \hat{\tau}}, Z$ is the number of the nearest neighbor or second-nearest neighbor sites, $p=\left(\mathbf{p}, i p_{m}\right), p^{\prime}=\left(\mathbf{p}^{\prime}, i p_{m}^{\prime}\right), \Sigma_{1 o}^{(h)}(k)$ and $\Sigma_{1 e}^{(h)}(k)$ are the symmetric and antisymmetric parts of $\Sigma_{1}^{(h)}(k)$, while the mean-field $(\mathrm{MF})$ spin Green's function, $D^{(0)-1}(p)=\left[\left(i p_{m}\right)^{2}-\omega_{\mathbf{p}}^{2}\right] / B_{\mathbf{p}}$, with $B_{\mathbf{p}}$, the MF spin excitation spectrum $\omega_{\mathbf{p}}$, and MF dressed holon excitation spectrum $\xi_{\mathbf{k}}$ have been given in Ref. [11].

Although $Z_{h F}(\mathbf{k})$ still is a function of $\mathbf{k}$, the wave vector dependence may be unimportant, since ARPES experiments have shown that around the nodal and antinodal points contain the essentials of the whole low energy quasiparticle spectrum $[2,3]$. Therefore in the following discussions, we only study the effects of the nodal and antinodal SC quasiparticle coherences on superconductivity, i.e., $Z_{h F}^{(N)}=$ $\left.Z_{h F}(\mathbf{k})\right|_{\mathbf{k}=[\pi / 2, \pi / 2]}$ at the nodal point, and $Z_{h F}^{(A)}=$ $\left.Z_{h F}(\mathbf{k})\right|_{\mathbf{k}=[\pi, 0]}$ at the antinodal point. On the other hand, for the understanding of the different influences of the SC quasiparticle coherence on the swave and d-wave SC-states, we consider, $\bar{\Delta}_{h Z}^{(s)}(\mathbf{k})=$ $\bar{\Delta}_{h Z}^{(s)} \gamma_{\mathbf{k}}^{(s)}$, with $\gamma_{\mathbf{k}}^{(s)}=\gamma_{\mathbf{k}}=\left(\cos k_{x}+\cos k_{y}\right) / 2$ for the s-wave pairing, and $\bar{\Delta}_{h Z}^{(d)}(\mathbf{k})=\bar{\Delta}_{h Z}^{(d)} \gamma_{\mathbf{k}}^{(d)}$, with $\gamma_{\mathbf{k}}^{(d)}=\left(\cos k_{x}-\cos k_{y}\right) / 2$ for the d-wave pairing. In this case, the dressed holon effective gap parameter and quasiparticle coherent weight satisfy the following two equations $[9,11]$,

$$
\begin{aligned}
1 & =\left.\frac{1}{\bar{\Delta}_{h}^{(a)}} \frac{4}{N} \sum_{\mathbf{k}} \gamma_{\mathbf{k}}^{(a)} \Sigma_{2}^{(h)}(\mathbf{k}, \omega)\right|_{\omega=0}, \\
Z_{h F}^{(\alpha)-1} & =1-\left.\Sigma_{1 o}^{(h)}\left(\mathbf{k}_{\alpha}, \omega\right)\right|_{\omega=0},
\end{aligned}
$$

respectively, where $a=s, d, \alpha=N, A$, and $\mathbf{k}_{\alpha}=$ $\mathbf{k}_{N}, \mathbf{k}_{A}$, with $\mathbf{k}_{N}=[\pi / 2, \pi / 2]$ and $\mathbf{k}_{A}=[\pi, 0]$. These two equations must be solved simultaneously with other self-consistent equations $[9,11]$, then all order parameters and chemical potential $\mu$ are determined by the self-consistent calculation.

With the help of the above discussions, we now can obtain the dressed holon pair gap parameter in terms of the holon off-diagonal Green's function (4) as $\Delta_{h}^{(a)}=(2 / N) \sum_{\mathbf{k}}\left[\gamma_{\mathbf{k}}^{(a)}\right]^{2} Z_{h F}^{(\alpha)} \bar{\Delta}_{h Z}^{(a)} \tanh \left[\frac{1}{2} \beta E_{h \mathbf{k}}\right] / E_{h \mathbf{k}}$.

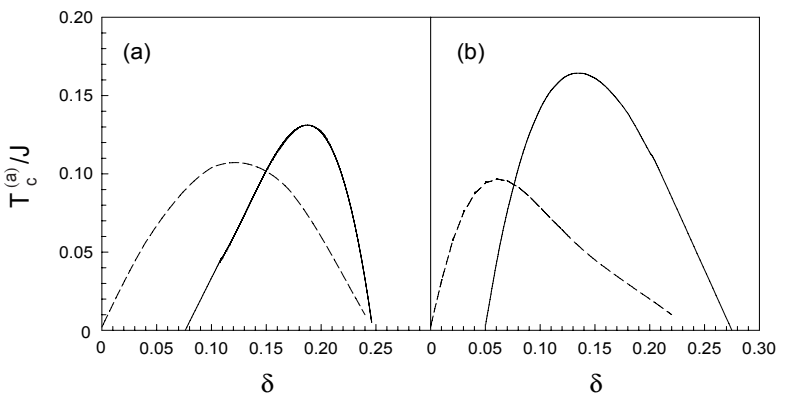

Fig. 1. The SC transition temperature $T_{c}^{(a)}$ as a function of doping in the d-wave (solid line) and s-wave (dashed line) cases for $t / J=2.5$ and $t^{\prime} / J=0.3$ with the SC quasiparticle coherence at (a) the nodal and (b) antinodal points.

This dressed holon pairing state originating from the kinetic energy terms by exchanging the spin excitations also leads to form the electron Cooper pairing state $[7,9]$, where the electron quasiparticle coherent weight and SC gap function are obtained from the electron diagonal and off-diagonal Green's functions $G\left(i-j, t-t^{\prime}\right)=\left\langle\left\langle C_{i \sigma}(t) ; C_{j \sigma}^{\dagger}\left(t^{\prime}\right)\right\rangle\right\rangle$ and $\Gamma^{\dagger}\left(i-j, t-t^{\prime}\right)=\left\langle\left\langle C_{i \uparrow}^{\dagger}(t) ; C_{j \downarrow}^{\dagger}\left(t^{\prime}\right)\right\rangle\right\rangle$, which are the convolutions of the spin Green's function and dressed holon diagonal and off-diagonal Green's functions, respectively, and reflect CSR [14]. This CSR transfers the dressed holon BCS type diagonal and off-diagonal Green's functions (3) and (4) into the corresponding electron BCS type diagonal and off-diagonal Green's functions, then the nature of the SC quasiparticle coherence is described by the simple BCS formalism [11], although the pairing mechanism is driven by the kinetic energy by exchanging the spin excitations. Following our previous discussions $[9,11]$, we can obtain $G(k)$ and $\Gamma^{\dagger}(k)$, then the electron quasiparticle coherent weight and effective SC gap parameter are evaluated as $Z_{F}^{(\alpha)} \approx Z_{h F}^{(\alpha)} / 2$ and $\bar{\Delta}^{(a)} \approx-\chi_{1} \bar{\Delta}_{h}^{(a)}$, respectively.

As we $[7,9]$ have shown that the SC transition temperature $T_{c}^{(a)}$ occurring in the case of the SC gap parameter $\Delta^{(a)}=0$ is identical to the dressed holon pair transition temperature occurring in the case of the dressed holon pairing gap parameter $\Delta_{h}^{(a)}=0$. In Fig. 1, we plot the SC transition temperature $T_{c}^{(a)}$ as a function of doping in the d-wave (solid line) and s-wave (dashed line) cases for $t / J=2.5$ and $t^{\prime} / J=0.3$ with the SC quasiparticle coherence at (a) the nodal and (b) antinodal points. It is shown that for the s-wave symmetry, the maximal SC transition temperature $\mathrm{T}_{c}^{(s)}$ occurs around a particular doping concentration, and then decreases in both 


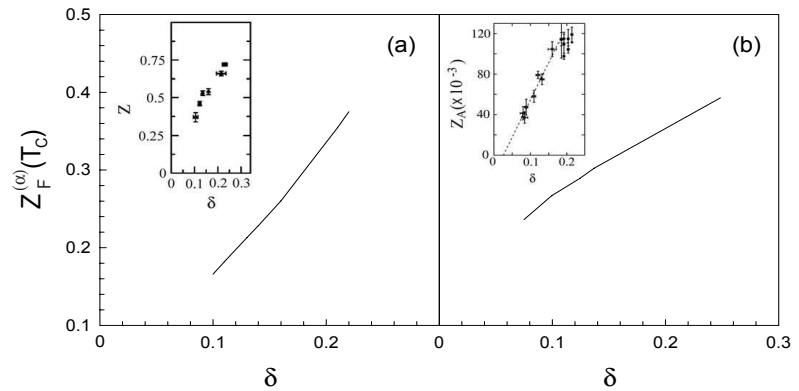

Fig. 2. (a) The nodal and (b) antinodal quasiparticle coherent weights $Z_{F}^{(\alpha)}\left(T_{c}\right)$ as a function of doping for $t / J=2.5$ and $t^{\prime} / t=0.3$. Inset: the corresponding experimental results of the nodal [6] and antinodal [5] quasiparticle coherent weights.

lower doped and higher doped regimes, while for the d-wave symmetry, the maximal SC transition temperature $\mathrm{T}_{c}^{(d)}$ occurs around the optimal doping, and then decreases in both underdoped and overdoped regimes. However, the s-wave SC transition temperature is heavily suppressed by the antinodal quasiparticle coherence, while the d-wave SC transition temperature is enhanced. Since the experimental results $[2,4,5]$ have shown that the SC-state in doped cuprates has the d-wave symmetry in a wide range of doping, then in this sense, the antinodal quasiparticle coherence plays a more crucial role for superconductivity. These results also are consistent with the experimental evidence that the most contributions of the electronic states for cuprate superconductors come from the antinodal point $[2,3]$.

The essential physics of superconductivity in the present case is the same as that in our previous discussions $[9,11]$. The antisymmetric part of the selfenergy $\Sigma_{1 o}^{(h)}(\mathbf{k})$ (then $Z_{F}^{(\alpha)}$ ) describes the dressed holon (then electron) quasiparticle coherence, and therefore $Z_{F}^{(\alpha)}$ is closely related to the SC quasiparticle density, while the self-energy $\Sigma_{2}^{(h)}(\mathbf{k})$ describes the effective dressed holon (then electron) pairing gap function. In particular, $Z_{F}^{(\alpha)}$ is doping dependent. To show this point clearly, we plot (a) the nodal and (b) antinodal SC quasiparticle coherent weights $Z_{F}^{(\alpha)}\left(T_{c}\right)$ as a function of doping for $t / J=2.5$ and $t^{\prime} / t=0.3$ in Fig. 2 in comparison with the corresponding experimental results of the nodal [6] and antinodal [5] SC quasiparticle coherent weights (inset). As seen from Fig. $2, Z_{F}^{(\alpha)}$ grows linearly with doping, i.e., $Z_{F}^{(\alpha)} \propto \delta$, which together with the SC gap parameter $[9,11]$ show that only $\delta$ number of the coherent doped carriers are recovered in the SCstate, consistent with the picture of a doped Mott insulator with $\delta$ holes [10]. Since the SC-order is established through an emerging SC quasiparticle [5], therefore the SC-order is controlled by both SC gap function and quasiparticle coherence, and is reflected explicitly in the self-consistent equations (7) and (8), this leads to that the SC transition temperature increases with increasing doping in the underdoped regime, and reaches a maximum in the optimal doping, then decreases in the overdoped regime.

In summary, we have discussed the interplay between superconductivity and the nodal and antinodal SC quasiparticle coherences in cuprate superconductors under the kinetic energy driven SC mechanism. It is shown that the s-wave SC transition temperature is heavily suppressed by the antinodal SC quasiparticle coherence, while the d-wave $\mathrm{SC}$ transition temperature is enhanced, therefore the antinodal SC quasiparticle coherence plays a more crucial role for superconductivity.

This work was supported by the National Natural Science Foundation of China under Grant Nos. 10125415 and 90403005, and the Grant from the Ministry of Science and Technology of China under Grant No. 2006CB601002.

\section{References}

[1] M.A.Kastner et al., Rev. Mod. Phys. 70 (1998) 897.

[2] A. Damascelli et al., Rev. Mod. Phys. 75 (2003) 475.

[3] J. Fink et al., cond-mat/0512307

[4] H. Matsui et al., Phys. Rev. Lett. 90 (2003) 217002.

[5] H. Ding et al., Phys. Rev. Lett. 87 (2001) 227001; D.L. Feng et al., Science 289 (2000) 277.

[6] P.D. Johnson et al., Phys. Rev. Lett. 87 (2001) 177007.

[7] Shiping Feng, Phys. Rev. B 68 (2003) 184501.

[8] Shiping Feng et al., J. Phys. Condens. Matter 16 (2004) 343; Mod. Phys. Lett. B 17 (2003) 361.

[9] Shiping Feng et al., Physica C 436 (2006) 14.

[10] P.W. Anderson, Science 235 (1997) 1196.

[11] Shiping Feng et al., Phys. Lett. A 350 (2006) 138; Huaiming Guo et al., cond-mat/0602652

[12] Z.X. Shen et al., Phys. Rev. Lett. 70 (1993) 1553; H. Ding et al., Phys. Rev. B 54 (1996) R9678.

[13] G.M. Eliashberg, Sov. Phys. JETP 11 (1960) 696; D.J. Scalapino et al., Phys. Rev. 148 (1966) 263.

[14] P.W. Anderson, Phys. Rev. Lett. 67 (1991) 2092; Science 288 (2000) 480; Physica C 341-348 (2000) 9. 